\title{
Biswapped Networks and Their Topological Properties
}

\author{
Wenjun Xiao ${ }^{1}$, Weidong Chen ${ }^{1,2}$, Mingxin $\mathrm{He}^{1}$, Wenhong Wei ${ }^{1}$, Behrooz Parhami ${ }^{3}$ \\ ${ }^{1}$ Department of Computer Science \\ South China University of Technology, Guangzhou 510641, P.R.China \\ ${ }^{2}$ Department of Computer Science \\ South China Normal University, Guangzhou 510631, P.R. China \\ ${ }^{3}$ Department of Electrical and Computer Engineering \\ University of California, Santa Barbara, CA 93106-9560, USA \\ E-mail: wjxiao@scut.edu.cn
}

\begin{abstract}
In this paper, we propose a new class of interconnection networks, called "biswapped networks" (BSNs). Each BSN is built of $2 n$ copies of some n-node basis network using a simple rule for connectivity that ensures its regularity, modularity, fault tolerance, and algorithmic efficiency. In particular, if the basis network is a Cayley digraph then so is the resulting BSN. Our proposed networks provide a systematic construction strategy for large, scalable, modular, and robust parallel architectures, while maintaining many desirable attributes of the underlying basis network that comprises its clusters. We show how key parameters of a BSN are related to the corresponding parameters of its basis network and obtain a number of results on internode distances, Hamiltonian cycles, and node-disjoint paths. We also discuss the relationship between BSNs and swapped or OTIS networks.
\end{abstract}

\section{Introduction}

The fact that Cayley (di)graphs and coset graphs are excellent models for interconnection networks, studied in connection with parallel processing and distributed computation, is widely acknowledged [1], [2], [5]. Many well-known interconnection networks are Cayley (di)graphs or coset graphs. For example, hypercube, butterfly, cube-connected cycles and honeycomb networks are Cayley graphs, while de Bruijn and shuffle-exchange networks are coset graphs [5], [8], [11], [12], [13], [14].

Much work on interconnection networks can be categorized as ad hoc design and evaluation. Typically, a new interconnection scheme is suggested and shown to be superior to some previously studied network(s) with respect to one or more performance or complexity attributes. Whereas Cayley (di)graphs have been used to explain and unify interconnection networks with many ensuing benefits, much work remains to be done. As suggested by Heydemann [5], general theorems are lacking for Cayley digraphs and more group theory has to be exploited to find properties of Cayley digraphs.

In this paper, we propose a new class of interconnection networks called "biswapped networks" (BSNs) that are related to swapped or OTIS networks, previously proposed and studied by a number of researchers [4], [7], [10], [15]. A network in the new proposed class is built of $2 n$ copies of some $n$-node basis network using a simple rule for connectivity that ensures its regularity, modularity, fault tolerance, and algorithmic efficiency. In particular, if the basis network is a Cayley (di)graph then so is the resulting BSN. Thus, the work presented here yields a systematic method for the construction of large, scalable, modular, and robust parallel architectures, while maintaining many desirable attributes of the underlying basis network that comprises its clusters. We show how key parameters a BSN are related to the corresponding parameters of its basis network and obtain results related to internode distances, Hamiltonicity, and node-disjoint paths. We also discuss the relations between BSNs and swapped [15] or OTIS [7] networks.

Before proceeding further, we introduce some definitions and notations related to (di)graphs, Cayley (di)graphs in particular, and interconnection networks. For more definitions and basic results on graphs and groups we refer the reader to [3], for instance, and on interconnection networks to [6], [9].

A digraph $\Gamma=(V, E)$ is defined by a set $V$ of vertices and a set $E$ of arcs or directed edges. Because we are interested in the use of graphs as parallel processing interconnection networks, we use the terms "vertex" and "node" interchangeably. Similarly, "edge" and "link" are fully equivalent in this paper. The edge set $E$ is a subset of elements $(u, v)$ of $V \times V$. If the subset $E$ is symmetric, that is, $(u, v) \in E$ implies $(v, u) \in E$, we identify two opposite $\operatorname{arcs}(u, v)$ and $(v, u)$ by the undirected edge $[u, v]$ and the digraph $\Gamma$ becomes an undirected graph. When the type of graph is clear from the context, we may use $(u, v)$ instead of $[u, v]$.

Let $G$ be a finite group and $S$ a subset of $G$. The subset $S$ is said to be a generating set for $G$, and the elements of $S$ are called generators of $G$, if every element of $G$ can be expressed as a finite product of 
their powers. We also say that $G$ is generated by $S$. The Cayley digraph of the group $G$ and the subset $S$, denoted by $\operatorname{Cay}(G, S)$, has vertices that are elements of $G$ and arcs that are ordered pairs $(g, g s)$ for $g \in G, s \in$ $S$. If $S$ is a generating set of $G$ then we say that $\operatorname{Cay}(G$, $S$ ) is the Cayley digraph of $G$ generated by $S$. If $4 \notin S$ ( 1 denotes the identity element of $G$ ) and $S=S^{-1}$, then $\operatorname{Cay}(G, S)$ is a simple graph

Assume that $\Gamma$ and $\Sigma$ are two digraphs. The mapping $\phi$ of $V(\Gamma)$ to $V(\Sigma)$ is a homomorphism from $\Gamma$ to $\Sigma$ if for any $(u, v) \in E(\Gamma)$ we have $(\phi(u), \phi(v)) \in E(\Sigma)$. In particular, if $\phi$ is a bijection such that both $\phi$ and the inverse of $\phi$ are homomorphisms then $\phi$ is called an isomorphism of $\Gamma$ to $\Sigma$. Let $G$ be a finite group and $S$ a subset of $G$. Assume that $K$ is a subgroup of $G$ (denoted as $K \leq G$ ). Let ${ }^{G} /{ }_{K}$ denote the set of the right cosets of $K$ in $G$. The (right) coset graph of $G$ with respect to subgroup $K$ and subset $S$, denoted by $\operatorname{Cos}(G$, $K, S)$, is the digraph with vertex set ${ }^{G} / K$ such that there exists an $\operatorname{arc}\left(K g, \mathrm{Kg}^{\prime}\right)$ if and only if there exists $s \in S$ and $K g s=K g^{\prime}$.

The following basic theorem, which can be easily proven, is helpful in establishing some of our subsequent results [14].

Theorem 1. For $g \in G, S \subseteq G$, and $K \leq G$, the mapping $\phi: g \rightarrow K g$ is a homomorphism from $\operatorname{Cay}(G, S)$ to $\operatorname{Cos}(G, K, S)$.

Unless explicitly specified, all graphs in this paper are undirected graphs. The notation used throughout this paper is listed in Table I for ease of reference.
Let $\Omega$ be any digraph with the vertex set $V(\Omega)=\left\{h_{1}\right.$, $\left.h_{2}, \ldots, h_{n}\right\}$ and the arc set $E(\Omega)$. The biswapped interconnection network $B s w(\Omega)=\Sigma=(V(\Sigma), E(\Sigma))$ is a digraph with its vertex and edge sets specified as:

$V(\Sigma)=\{\langle 0, p, g\rangle,\langle 1, p, g\rangle \mid p, g \in V(\Omega)\}$

$E(\Sigma)=\left\{\left(\left\langle 0, p, g_{1}\right\rangle,\left\langle 0, p, g_{2}\right\rangle\right),\left(\left\langle 1, p, g_{1}\right\rangle,\left\langle 1, p, g_{2}\right\rangle\right)\right.$

$\left.\mid\left(g_{1}, g_{2}\right) \in E(\Omega), p \in V(\Omega)\right\}$

$p\rangle) \mid p, g \in V(\Omega)\}$

$$
\cup\{(\langle 0, p, g\rangle,\langle 1, g, p\rangle),(\langle 1, p, g\rangle,\langle 0, g,
$$

Intuitively, the definition postulates $2 n$ clusters, each cluster being an $\Omega$ digraph: $n$ clusters, with nodes numbered $\langle 0$, cluster\#, node\# $\rangle$, form part 0 of the bipartite graph, and $n$ clusters constitute part 1, with associated node numbers $\langle 1$, cluster\#, node\# $\rangle$. Each cluster $p$ in either part of $\Sigma$ has the same internal connectivity as $\Omega$ (intracluster edges, forming the first set in the definition of $E(\Sigma)$ ). In addition, node $g$ of cluster $p$ in part $0 / 1$ is connected to node $p$ in cluster $g$ of part 1/0 (intercluster or swap edges in the second set in the definition for $E(\Sigma)$ ). The name "biswapped network" (BSN) arises from two defining properties of the network just introduced: when clusters are viewed as supernodes, the resulting graph of supernodes is a complete $2 n$-node bipartite graph, and the intercluster links connect nodes in which the cluster number and the node number within cluster are interchanged or swapped.

As an example, when $\Omega=C_{4}$ (the undirected cycle of order 4) constitutes the basis graph, Fig. 1 depicts the resulting $B s w\left(C_{4}\right)$. Part 0 of the network is drawn at the top and part 1 at the bottom, with clusters $0-3$ positioned from left to right.

\section{Definitions and Basic Properties}

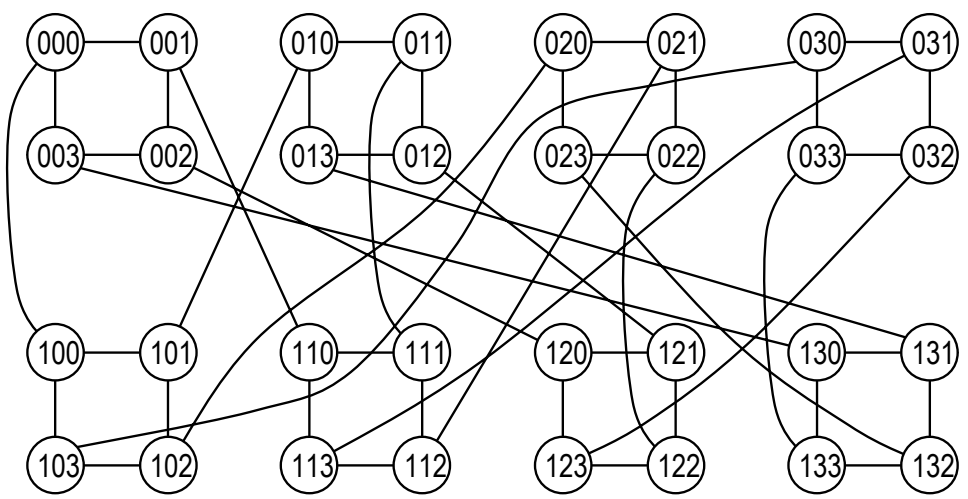

Fig. 1. An example 32-node biswapped network using the basis graph $\Omega=C_{4}$. Each line represents two directed edges in opposite directions.

To avoid clutter, the node index $\langle i, p, g\rangle$ is shown as ipg.

We could continue our presentation with directed networks, deriving results for undirected networks as special cases. However, because parallel processing interconnection networks are usually undirected, we focus on undirected graphs in the rest of this paper. For undirected biswapped networks, the definition of the edge set is simplified to:

$$
E(\Sigma)=\left\{\left(\left\langle 0, p, g_{1}\right\rangle,\left\langle 0, p, g_{2}\right\rangle\right),\left(\left\langle 1, p, g_{1}\right\rangle,\left\langle 1, p, g_{2}\right\rangle\right)\right.
$$

$\left.\mid\left(g_{1}, g_{2}\right) \in E(\Omega), p \in V(\Omega)\right\}$ $\cup\{(\langle 0, p, g\rangle,\langle 1, g, p\rangle) \mid p, g \in V(\Omega)\}$

We need a few more notational conventions in what follows. For any graph $\Gamma$, the number of its nodes is denoted as $|\Gamma|$. The degree of a node $g$ in $\Gamma$ is $\operatorname{deg}_{\Gamma}(g)$. The distance between nodes $g_{1}$ and $g_{2}$ in $\Gamma$ is given by $\operatorname{dist}_{\Gamma}\left(g_{1}, g_{2}\right)$. The diameter of $\Gamma$, that is, the maximum distance between any two nodes in $\Gamma$, is $D(\Gamma)$. We first prove the following results on the basic parameters of $\Sigma=B s w(\Omega)$. 
Theorem 2. Let $\Sigma=B s w(\Omega)$. Then:

(1) $|\Sigma|=2|\Omega|^{2}$

(2) $\operatorname{deg}_{\Sigma}(\langle i, p, g\rangle)=\operatorname{deg}_{\Omega}(g)+1$

(3) $\operatorname{dist}_{\Sigma}\left(\left\langle i, p_{1}, g_{1}\right\rangle,\left\langle i, p_{2}, g_{2}\right\rangle\right)$ equals $\operatorname{dist}_{\Omega}\left(g_{1}, g_{2}\right)$ if $p_{1}=p_{2}$; otherwise, it equals $\operatorname{dist}_{\Omega}\left(p_{1}, p_{2}\right)+\operatorname{dist}_{\Omega}\left(g_{1}\right.$, $\left.g_{2}\right)+2$

(4) $\operatorname{dist}_{\Sigma}\left(\left\langle 0, p_{1}, g_{1}\right\rangle,\left\langle 1, p_{2}, g_{2}\right\rangle\right)=\operatorname{dist}_{\Omega}\left(p_{1}, g_{2}\right)+$ $\operatorname{dist}_{\Omega}\left(p_{2}, g_{1}\right)+1$

Proof. Omitted.

Corollary 1. The diameter of $B \operatorname{sw}(\Omega)=\Sigma$ is related to the diameter of the basis network $\Omega$ by the equality $D(\Sigma)=2 D(\Omega)+2$.
Proof. Omitted.

Based on Theorem 2, we can easily obtain a routing algorithm for a biswapped network, assuming the availability of a routing algorithm for the basis graph $\Omega$. Assume that the latter routing algorithm is a distributed one, using the local function $\operatorname{next}_{\Omega}\left(g_{1}, g_{2}\right)$ to obtain the first intermediate node in the routing path from $g_{1}$ to $g_{2}$. Then, the algorithm shown in Fig. 2 can be used to derive the first intermediate node on a shortest routing path from node $\left\langle i, p_{1}, g_{1}\right\rangle$ to node $\left\langle j, p_{2}\right.$, $\left.g_{2}\right\rangle$ in $\operatorname{Bsw}(\Omega)=\Sigma$.

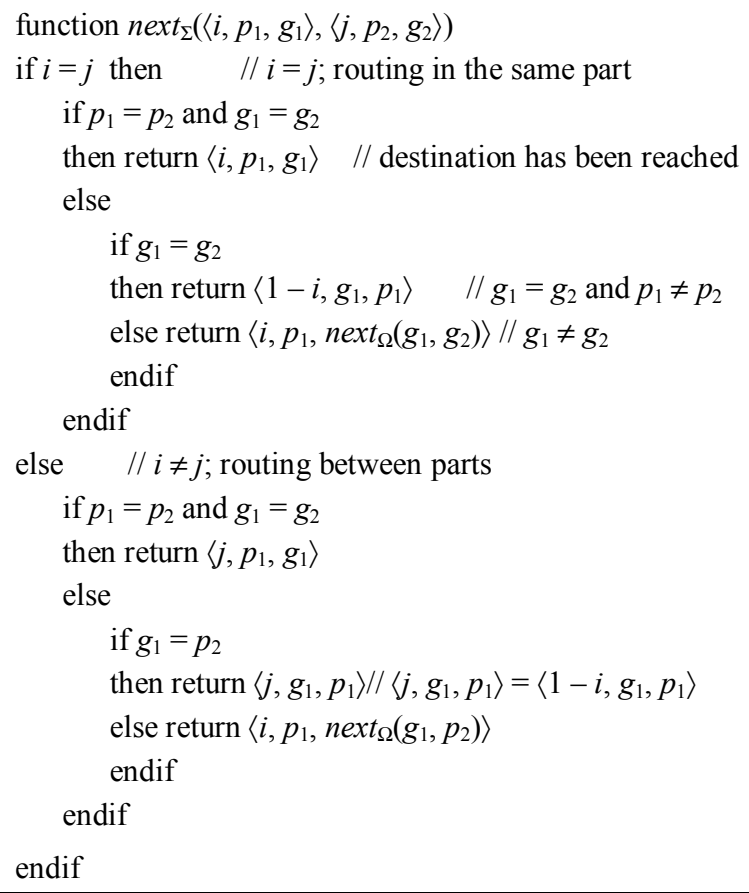

Fig. 2. Optimal routing function for a biswapped network $B s w(\Omega)$ based on the optimal routing function $\operatorname{Next}_{\Omega}\left(g_{1}, g_{2}\right)$ for its basis network $\Omega$.

It is readily seen that the routing function $N e x t_{\Sigma}$ defined above is optimal, that is, it leads to shortestpath routing, if the routing function $N e x t_{\Omega}$ is optimal.

\section{Hamiltonicity and Disjoint Paths}

A Hamiltonian cycle of a graph is a cycle that visits each node exactly once. A graph is Hamiltonian if it contains a Hamiltonian cycle. Hamiltonicity is a useful property for interconnection networks. One of our main results in this paper is that if the basis graph $\Omega$ is Hamiltonian, then so is the resulting biswapped network.

Theorem 3. If the basis graph $\Omega$ is Hamiltonian, then so is the graph $B s w(\Omega)=\Sigma$.

Proof. Let $\{0,1, \ldots, n-1,0\}$ represent a Hamiltonian cycle of the basis graph $\Omega$. Then, the following is a Hamiltonian cycle of the graph $\Sigma$ beginning and ending at node $\langle 0,0,0\rangle$ :

$\langle 0,0,0\rangle \rightarrow\langle 1,0,0\rangle \rightarrow\langle 1,0, n-1\rangle \rightarrow \ldots \rightarrow\langle 1,0,2\rangle$ $\rightarrow\langle 1,0,1\rangle \rightarrow\langle 0,1,0\rangle \rightarrow\langle 0,1, n-1\rangle \rightarrow \ldots \rightarrow\langle 0,1$, $2\rangle \rightarrow\langle 0,1,1\rangle \rightarrow\langle 1,1,1\rangle \rightarrow\langle 1,1,0\rangle \rightarrow \ldots \rightarrow\langle 1,1,3\rangle$ $\rightarrow\langle 1,1,2\rangle \rightarrow\langle 0,2,1\rangle \rightarrow\langle 0,2,0\rangle \rightarrow \ldots \rightarrow\langle 0,2,3\rangle \rightarrow$ $\langle 0,2,2\rangle \rightarrow \ldots \rightarrow\langle 1, n-2, n-2\rangle \rightarrow\langle 1, n-2, n-3\rangle \rightarrow$ $\ldots \rightarrow\langle 1, n-2,0\rangle \rightarrow\langle 1, n-2, n-1\rangle \rightarrow\langle 0, n-1, n-$ $2\rangle \rightarrow\langle 0, n-1, n-3\rangle \rightarrow \ldots \rightarrow\langle 0, n-1,0\rangle \rightarrow\langle 0, n-$ $1, n-1\rangle \rightarrow\langle 1, n-1, n-1\rangle \rightarrow\langle 1, n-1, n-2\rangle \rightarrow \ldots$ $\rightarrow\langle 1, n-1,1\rangle \rightarrow\langle 1, n-1,0\rangle \rightarrow\langle 0,0, n-1\rangle \rightarrow\langle 0,0, n$ $-2\rangle \rightarrow \ldots \rightarrow\langle 0,0,1\rangle \rightarrow\langle 0,0,0\rangle$

Figure 3 shows the constructed Hamiltonian cycle for a biswapped network.

We next consider the problem of node-disjoint paths between certain pairs of nodes in a biswapped network. We have the following. 


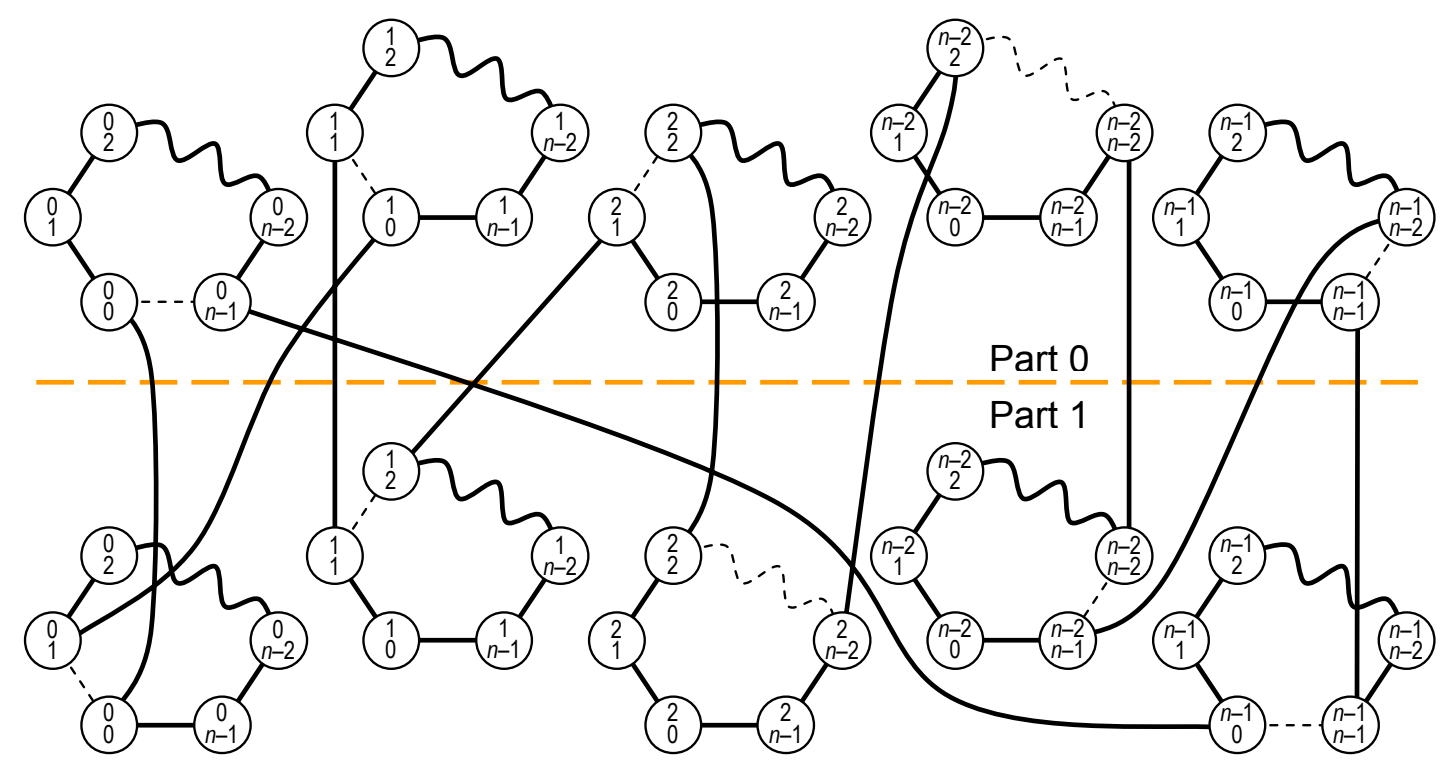

Fig. 3. A Hamiltonian cycle in a biswapped network whose basis graph $\Omega$ is Hamiltonian $(n=5)$.

Within each cluster, only links belonging to the cluster's Hamiltonian cycle are shown.

To avoid clutter, part numbers are not represented in node indices. Cluster index appears at the top and node index within cluster at the bottom inside the circles.

Theorem 4. If the basis graph $\Omega$ is connected, $p_{1} \neq p_{2}$, and $\operatorname{deg}_{\Omega}(g)=\delta$, then there are $\delta+1$ node-disjoint paths between the nodes $\left\langle i, p_{1}, g\right\rangle$ and $\left\langle i, p_{2}, g\right\rangle$ in $\operatorname{Bsw}(\Omega)=\Sigma$.

Proof. Omitted.

Note that Theorem 4 is quite strong in that it requires merely that $\Omega$ be connected. The following result shows that if $\Omega$ is $\delta$-connected, then $\operatorname{Bsw}(\Omega)$ will be ( $\delta$ $+1)$-connected.

Theorem 5. If the basis graph $\Omega$ is connected, and if $\langle i$, $\left.p_{1}, g_{1}\right\rangle$ and $\left\langle i, p_{2}, g_{2}\right\rangle$ are two nodes in $\operatorname{Bsw}(\Omega)=\Sigma$, with $g_{1} \neq g_{2}$, such that there are $\delta$ node-disjoint paths between $g_{1}$ and $g_{2}$ in $\Omega$, then there are $\delta+1$ nodedisjoint paths between the nodes $\left\langle i, p_{1}, g_{1}\right\rangle$ and $\left\langle i, p_{2}\right.$, $\left.g_{2}\right\rangle$ in $\Sigma$.

Proof. Omitted.

\section{Relationship with Swapped Networks}

We begin by defining a swapped network. For the sake of regularity, our definition is slightly different from that in [10] and [15].

Let $\Omega$ be any digraph with the vertex set $V(\Omega)=\left\{h_{1}\right.$, $\left.h_{2}, \ldots, h_{n}\right\}$ and the edge set $E(\Omega)$. The swapped interconnection network based on $\Omega$, that is, $\operatorname{Sw}(\Omega)=\Gamma$ $=(V(\Gamma), E(\Gamma))$, is a graph with its vertex and edge sets specified as:

$$
\begin{aligned}
& V(\Gamma)=\{\langle p, g\rangle \mid p, g \in V(\Omega)\} \\
& E(\Gamma)=\left\{\left(\left\langle p, g_{1}\right\rangle,\left\langle p, g_{2}\right\rangle\right) \mid\left(g_{1}, g_{2}\right) \in E(\Omega), p \in\right.
\end{aligned}
$$$$
V(\Omega)\} \cup\{(\langle p, g\rangle,\langle g, p\rangle) \mid p, g \in V(\Omega)\}
$$

Note that the difference between this definition and that in [10] and [15] is that the case $p=g$ is not excluded from the second set in the definition of $E(\Gamma)$; in other words, here we postulate that the swap link associated with a node $\langle p, p\rangle$ in $S w(\Omega)$ is a self-loop, whereas in the original definition of [10] and [15], node $\langle p, p\rangle$ lacks a swap link and thus has a node degree that is one less than that of node $\langle p, g\rangle$ with $p \neq$ $g$. The swapped network based on a regular $n$-node, degree- $d$ network $\Omega$ has $n^{2}$ nodes of degree $d+1$.

Because the class of Cayley graphs exhibits many desirable properties and also includes a significant fraction of all networks that have been found useful in parallel processing, we next consider biswapped networks built from basis networks that are Cayley graphs. It would indeed be quite an accomplishment if we could establish that biswapped networks thus formed are themselves Cayley graphs, because this would mean that certain desirable properties of the basis network are inherited by the composite biswapped network (we have already shown that Hamiltonicity is in fact transferred from the basis network to the biswapped network). In what follows, we will show that a Cayley-graph basis network does indeed lead to a biswapped network that is a Cayley graph.

Let $H$ be a finite group and $S$ a generator set of $H$, with $\Omega=\operatorname{Cay}(H, S)$ and $H \times H$ the direct product of the group $H$ and itself. Let $G=(H \times H)\langle t\rangle=\langle t\rangle(H \times H)$ be a semidirect product of the group $H \times H$ by the cyclic group $\langle t\rangle$, where $t$ is an element of order 2, and $t(p, g) t$ $=(g, p)$ for any $p, g \in H$. Let $S^{\prime}=\{(1, s) \mid s \in S\} \subseteq H$ $\times H$ and $T=S^{\prime} \cup\{t\}$. Suppose that $\Delta=\operatorname{Cos}(G,\langle t\rangle, T)$ is the coset graph of the group $G$ with respect to the 
subgroup $\langle t\rangle$ and the generator set $T$. Then we have the following result.

Theorem 5. The graph $\Delta$ defined in the preceding paragraph is isomorphic to the swapped network $\operatorname{Sw}(\Omega)=\Gamma$.

Proof. The correspondence $\phi:\langle t\rangle(p, g) \rightarrow\langle p, g\rangle$ is a mapping from $\Delta$ to $\Gamma$. Moreover, for $p, g, q, h \in H$, $(\langle t\rangle(p, g),\langle t\rangle(q, h))$ is an edge of the graph $\Delta$ if and only if either there is an element $s \in S$ such that $h=g s$ and $p=q$ or $\langle t\rangle(q, h)=\langle t\rangle(p, g) t=\langle t\rangle(g, p)$. This is equivalent to saying that $(\langle p, g\rangle,\langle q, h\rangle)$ is an edge of the graph $\Gamma$. Hence, we have $\Delta \cong \Gamma$.

Let us consider a concrete example to illustrate the result of Theorem 5. Let $\Omega=\operatorname{Cay}\left(Z_{n}, S\right)$, with $S=$ $\{ \pm 1\}$. Then, $G=\left(Z_{n} \times Z_{n}\right)\langle t\rangle=\langle t\rangle\left(Z_{n} \times Z_{n}\right)$, with $S^{\prime}=$
$\{(0, \pm 1)\}$ and $T=S^{\prime} \cup\{t\}$. Let $\Delta=\operatorname{Cos}(G,\langle t\rangle, T)$. Then, $\Delta \cong S w(\Omega)=\Gamma$ through the mapping $\phi:\langle t\rangle(p, g)$ $\rightarrow\langle p, g\rangle$.

By Theorem 5, there is a homomorphism from the biswapped network $B s w(\Omega)$ to the swapped network $\operatorname{Sw}(\Omega)$. This is depicted in Fig. 4 for an example network.

Let $\Psi=\operatorname{Cay}(G, T)$ be the Cayley graph of the group $G$ and the generator set $T$. Then we may prove the following result in a manner similar to Theorem 5.

Theorem 6. The graph $\Psi=C a y(G, T)$, with $G$ and $T$ as defined in the paragraph preceding Theorem 5 , is isomorphic to the biswapped network $B s w(\Omega)=\Sigma$.

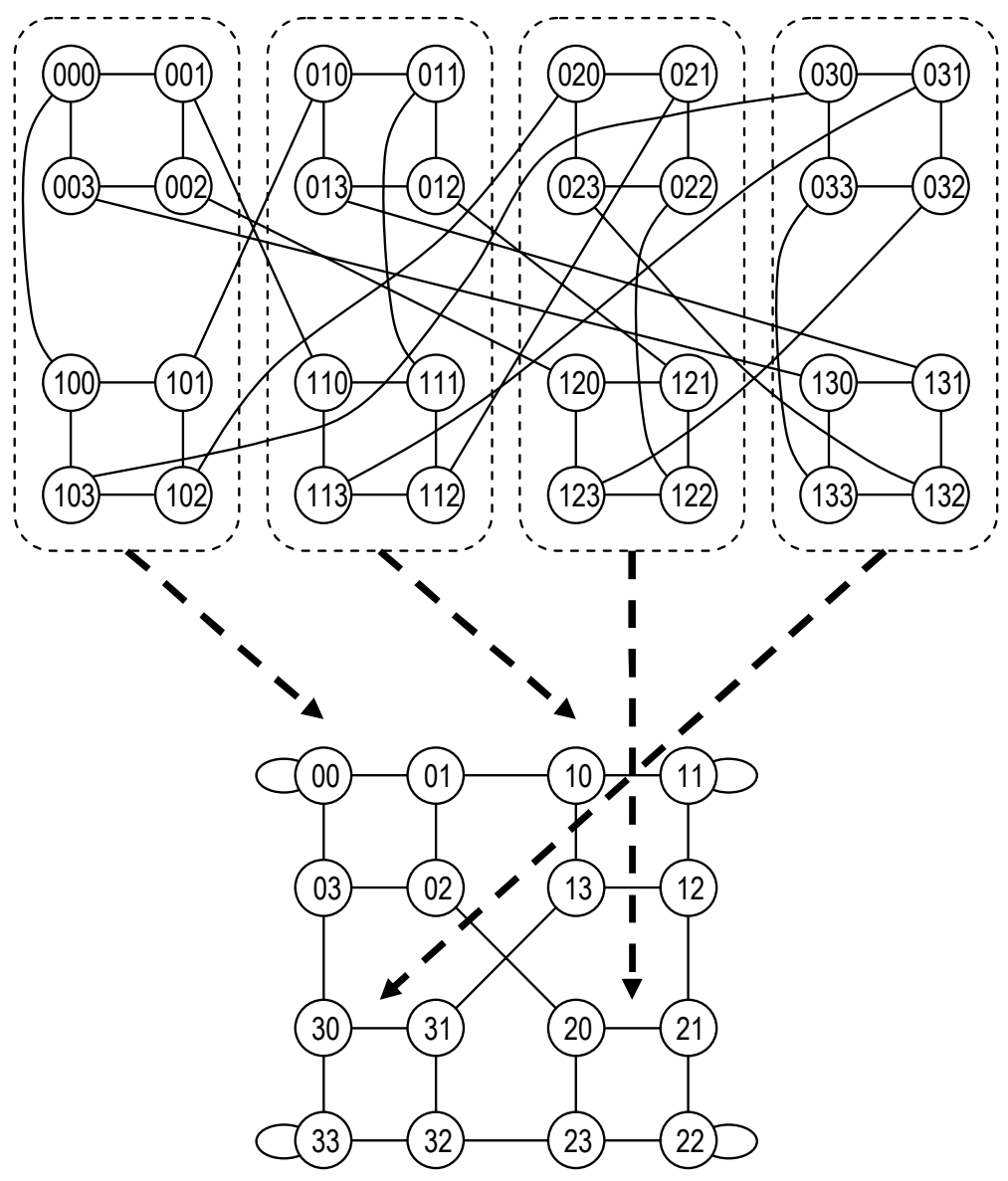

Fig. 4. The 32-node biswapped network with the basis graph $\Omega=C_{4}$ is homomorphic to the 16-node swapped network using the same basis graph, with the latter being identical to its counterpart in [10] if all the self-loops are removed.

\section{Conclusions}

In this paper, we have provided a number of general results on a new class of interconnection networks called "biswapped networks." A biswapped network is composed of $2 n$ copies of an $n$-node basis network using a simple rule for connectivity that ensures its regularity, modularity, fault tolerance, and algorithmic efficiency. In particular, if the basis network is a Cayley (di)graph then so is the associated biswapped network. Biswapped networks provide us with a systematic method for constructing large, scalable, modular, and robust parallel architectures, while 
maintaining many desirable attributes of the underlying basis network that comprise their clusters. We showed how key parameters of a biswapped network are related the corresponding parameters of its basis network and obtained results related to distances between nodes, Hamiltonicity, and node-disjoint paths. We also discussed the relations between the new networks and the known swapped or OTIS networks.

Results in this paper bring some closure to the topic of swapped or OTIS networks, which as previously defined (or even with the modified form introduced at the beginning of Section 4) lack full symmetry. Biswapped networks are completely symmetric and offer twice as many nodes as the corresponding swapped networks with the same node degree and with a unit increase in network diameter. Because of the generality of our theorems, we expect that they will find many more applications than those discussed here.

We are currently investigating the applications of our method to the problems related to routing and average internode distance in certain subclasses of our networks. These results, along with potential applications in the following areas will be reported in future:

- Load balancing and congestion control

- Scheduling and resource allocation

- Fault tolerance and graceful degradation

These constitute important practical problems in the design, evaluation, and efficient operation of parallel and distributed computer systems.

\section{Acknowledgment}

Research of the first four authors was supported by the Natural Science Foundation of China and Guangdong Province (No.04020130).

\section{References}

[1] S. B. Akers and B. Krishnamurthy, "A Group Theoretic Model for Symmetric Interconnection Networks," IEEE Trans. Computers, Vol. 38, pp. 555-566, 1989.

[2] F. Annexstein, M. Baumslag, and A. L. Rosenberg, "Group Action Graphs and Parallel Architectures," SIAM J. Computing, Vol. 19, pp. 544-569, 1990.

[3] N. Biggs, Algebraic Graph Theory, Cambridge University Press, 1993.
[4] K. Day and A. Al-Ayyoub, "Topological Properties of OTIS-Networks," IEEE Trans. Parallel and Distributed Systems, Vol. 13, No. 4, pp. 359-366, April 2002.

[5] M. Heydemann, "Cayley Graphs and Interconnection Networks," in Graph Symmetry: Algebraic Methods and Applications, 1997, pp. 167-224.

[6] F. T. Leighton, Introduction to Parallel Algorithms and Architectures: Arrays, Trees, Hypercubes, Morgan Kaufmann, 1992.

[7] G. Marsden, P. Marchand, P. Harvey, and S. Esener, "Optical Transpose Interconnection System Architectures," Optics Letters, Vol. 18, No. 13, pp. 1083-1085, 1998.

[8] F. G. Nocetti, I. Stojmenovic, and J. Zhang, "Addressing and Routing in Hexagonal Networks with Applications for Tracking Mobile Users and Connection Rerouting in Cellular Networks," IEEE Trans. Parallel and Distributed Systems, Vol. 13, pp. 963-971, 2002.

[9] B. Parhami, Introduction to Parallel Processing: Algorithms and Architectures, Plenum, 1999.

[10] B. Parhami, "Swapped Interconnection Networks: Topological, Performance, and Robustness Attributes," J. Parallel and Distributed Computing, Vol. 65, pp. 1443-1452, 2005.

[11] B. Parhami and D. M. Kwai, "A Unified Formulation of Honeycomb and Diamond Networks," IEEE Trans. Parallel and Distributed systems, Vol. 12, pp. 74-80, 2001.

[12] I. Stojmenovic, "Honeycomb Networks: Topological Properties and Communication Algorithms," IEEE Trans. Parallel and Distributed Systems, Vol. 8, pp. 1036-1042, 1997.

[13] W. J. Xiao and B. Parhami, "Some Mathematical Properties of Cayley Digraphs with Applications to Interconnection Network Design," International J. Computer Mathematics, Vol. 82, pp. 521-528, 2005.

[14] W. J. Xiao and B. Parhami, "Further Mathematical Properties of Cayley Digraphs Applied to Hexagonal and Honeycomb Meshes," Discrete Applied mathematics, to appear.

[15] C.-H. Yeh and B. Parhami, "Swapped Networks: Unifying the Architectures and Algorithms of a Wide Class of Hierarchical Parallel Processors," Proc. International Conf. Parallel and Distributed Systems, June 1996, pp. 230-237. 